\title{
GENDER DIFFERENCES IN THE GENETIC AND ENVIRONMENTAL DETERMINANTS OF ADOLESCENT DEPRESSION
}

\author{
Monica Uddin, Ph.D., ${ }^{1 *}$ Karestan C. Koenen, Ph.D., ${ }^{2}$ Regina de los Santos, M.S., ${ }^{1}$ Erin Bakshis, M.P.H., ${ }^{1}$ \\ Allison E. Aiello, Ph.D., ${ }^{1}$ and Sandro Galea, M.D., DrPH. ${ }^{3}$
}

Background: The well-documented gender differences in the risk for depression may be explained by genetic factors, by different responses to social context, or by a combination of both. We sought to assess whether there were gender differences in the longitudinal associations between serotonin transporter promoter (5-HTTLPR) genotype and depressive symptoms in adolescents, and whether macrosocial context plays a role in explaining any observed differences. Methods: Using data from a nationally representative survey of adolescents, we applied multilevel mixed models to assess, separately for adolescent males and females (a) the relation between 5-HTTLPR genotype and depressive symptoms and (b) the interaction of county-level deprivation and 5-HTTLPR genotype in models predicting depressive symptoms. All models adjusted for age and other covariates. Results: Among females $(n=560)$, main effects models showed an association between the sl genotype and lowered risk of depressive symptoms $(b=-.18, P=.03)$. Among males $(n=524)$, interaction models showed an association between sl genotype and lowered risk of depressive symptoms in deprived counties only $(b=-.32, P=.04)$. Conclusions: In adolescent females, the 5-HTTLPR sl genotype confers protection against depressive symptoms independent of county-level social context, whereas in adolescent males, protection by the same genotype is conferred only within the context of countylevel deprivation. Future work should aim to understand bow genetic and macrosocial factors jointly shape risk for mental illness, and how these factors shape gender differences in mental illness. Depression and Anxiety 27:658-666, 2010. (C) 2010 Wiley-Liss, Inc.

Key words: social epidemiology; serotonin transporter promoter; geneenvironment interaction; macrosocial environment

\footnotetext{
${ }^{1}$ Department of Epidemiology, University of Michigan School of Public Health, Ann Arbor, Michigan

${ }^{2}$ Departments of Society, Human Development, and Health and Epidemiology, Harvard School of Public Health, Cambridge, Massachusetts

${ }^{3}$ Department of Epidemiology, Columbia University Mailman School of Public Health, New York, New York
}

Received for publication 22 December 2009; Revised 20 February 2010; Accepted 23 February 2010
The authors disclose the following financial relationships within the past 3 years: Contract grant sponsor: National Institute on Drug Abuse; Contract grant number: DA022720|DA022720-S1; Contract grant sponsor: National Insitute of Mental Health; Contract grant number: MH078152| MH070627IMH078928.

*Correspondence to: Monica Uddin, Department of Epidemiology, University of Michigan School of Public Health, 1415 Washington Heights, Ann Arbor, Ml 48109-2029. E-mail: uddinm@umich.edu

DOI 10.1002/da.20692

Published online 24 March 2010 in Wiley InterScience (www. interscience.wiley.com).

(C) 2010 Wiley-Liss, Inc. 


\section{INTRODUCTION}

G enetic influences on adolescent depression have been confirmed by both twin and adoption studies, with heritability estimates (i.e. the proportion of variation attributable to genetic factors) generally ranging from $30 \%$ to $50 \%$ (reviewed in ${ }^{[1]}$ ), a finding broadly comparable with estimates of major depressive disorder in adults. ${ }^{[2]}$ Notably, some studies have reported a greater genetic contribution to depression or depressive symptoms among boys versus girls and a more substantial effect of the common environment among girls versus boys ${ }^{[3,4]}$; others, however, have found genetic influences to be more important among adolescent girls. ${ }^{[5]}$ These conflicting results may be due in part to the fact that genetic influences on depression may only be evident under particular environmental conditions-i.e. that there may be Gene $\times$ Environment $(\mathrm{G} \times \mathrm{E})$ interactions, such that individuals of the same genotype may express different phenotypes depending on their surrounding social contexts. ${ }^{[6]}$

The gene encoding the serotonin transporter protein (SLC6A4) is the best studied in this regard. This protein serves many functions; however, its action has been particularly well-studied in the brain where it transports serotonin at synaptic terminals and other neuronal areas ${ }^{[7]}$ and serves to regulate emotional aspects of behavior. ${ }^{[8]}$ Commonly occurring repeat polymorphisms in the promoter $(5-H T T L P R)$ region of this gene have previously been associated with differential uptake of serotonin in in vitro studies. ${ }^{[9]}$ In humans, epidemiologic studies have reported $\mathrm{G} \times \mathrm{E}$ interactions between particular 5-HTTLPR alleles and depression, with the shorter, less transcriptionally active "s" allele being generally associated with greater risk for depression among individuals who have experienced maltreatment as children. ${ }^{[10]}$. However, attempts to replicate initial reports of these associations have been mixed and, in some cases, other 5-HTTLPR alleles and/or genotypes have been implicated in susceptibility to depression (reviewed in ${ }^{[11]}$ ). Most recently, a meta-analysis of 5-HTTLPR genotype, number of stressful life events (SLE), and potential interactions between the two concluded that only SLE showed evidence for a significant association with depression. ${ }^{[12]}$

Findings in adolescent samples have been particularly contradictory and suggest gender difference may be one explanation. The few studies published to date that focus on this age group have found evidence for increased risk for depression and depressive symptoms among carriers of the $l l$ genotype when combined with high levels of family adversity ${ }^{[13,14]}$ and among ss carriers when combined with a history of sexual abuse ${ }^{[15]}$ or high-risk backgrounds. ${ }^{[16]}$ Notably, a few studies have demonstrated interaction effects only when analyses were conducted stratified by gender, ${ }^{[16]}$ with some even identifying the same genotype as both a risk and protective factor against depression depending on the gender. ${ }^{[17]}$ The likelihood that the serotonergic system-to which the SLC6A4 locus belongs—operates differently in males and females is substantiated by evidence from animal models showing gender-specific differences in serotonin levels detected in the brain ${ }^{[18]}$ and blood. ${ }^{[19]}$ Examples such as these thus suggest that at least some of the heterogeneity in $\mathrm{G} \times \mathrm{E}$ interactions involving the 5-HTTLPR locus reported to date may be due to factors that interact differently according to gender and, possibly, developmental period. ${ }^{[11,20]}$

An additional consideration in $\mathrm{G} \times \mathrm{E}$ investigations is the type of environmental exposure being assessed. Although the predominant theme in these studies has been to measure the number of SLE ${ }^{[12]}$ or presence/ absence of maltreatment during childhood, ${ }^{[10,21,22]}$ evidence from both the non-genetic and genetic literature suggests that the macrosocial environment may also influence the risk for depression or depressive symptoms and that this effect is modified by gender. In the well-known "Moving to Opportunity" study, [23] adolescent males who moved to less impoverished neighborhoods were significantly less likely to report anxious/depressive problems than adolescent control males who did not move; a similar difference was not detected in adolescent females $\left({ }^{[23]}\right.$; but see ${ }^{[24]}$ for an alternate analysis). On the genetic side, 5-HTTLPR $\times \mathrm{E}$ interaction effects have been detected for adolescent boys according to their residence in public versus privately owned housing; no such interaction was detected for adolescent females ${ }^{[17]}$ although other variables, such as traumatic conflicts in the family, did show significant $\mathrm{G} \times \mathrm{E}$ interactions exclusively in this group. Emerging evidence thus suggests that gender differences in both the genetic and environmental determinants of depression and depressive symptoms exist in adolescent populations.

To test this hypothesis, we investigated the longitudinal association among 5-HTTLPR genotype, county-level deprivation, and depressive symptom scores in adolescent males and females sampled in the National Longitudinal Study of Adolescent Health (Add Health). Our goals were to test for the effect of 5-HTTLPR genotype on depressive symptom scores separately in adolescent boys and girls and to test whether any observed 5-HTTLPR effects were modified by macrosocial conditions in a large, community-based epidemiologic sample.

\section{MATERIALS AND METHODS}

\section{SAMPLE}

The data for this analysis was drawn from the National Longitudinal Study of Adolescent Health (Add Health), a nationally representative, school-based sample of over 90,000 adolescents in grades 7-12, initially sampled in 1994-1995 in the United States and followed for three subsequent waves. A subsample $(\sim 20,745)$ of participants from the in-school portion of the study was selected to participate in an additional, 90-min in-home interview during Wave I, which provided the primary data source for the analyses reported in this study. In 2002, during Wave III, DNA samples were collected 
from a subsample of siblings $(n=2,574)$ who had participated in the in-home interview portion of the study. The in-home and genetic data are part of the restricted use/contractual AddHealth data set ${ }^{[25]}$ and IRB approval to work with this data set was secured. More detail regarding the design and data availability for the genetic component of AddHealth is available elsewhere. ${ }^{[26]}$

The sample for our analysis draws on 1,084 individuals from the sibling subsample who provided DNA, belonged to a same sex sibling cluster of the same sibling type, and for whom there was a complete set of data available for each sibling in the cluster for each of the measures included in our models (described below). The analytic sample did not differ from the excluded sample with respect to genotype, measure of county-level deprivation, or depressive symptom scores, i.e. the main variables in the study.

\section{MEASURES}

Individual- and family-level health indicators. Depressive symptom scores were obtained using a shortened, 17-item version of the Center for Epidemiological Studies Depression Scale (CES-D), ${ }^{[27]}$ based on the CES-D questions that were posed in the AddHealth Feelings Scale during the in-home interviews conducted during Wave I (April-December 1995) and II (April-August 1996). The internal consistency from Wave I and II were 0.86 and 0.87 , respectively. Responses to the 17 questions were ordinal, ranging from 1 (never or rarely) to 4 (most or all of the time) and were summed for use as the outcome variable in all analyses, with higher scores indicative of more depressive symptoms. Respondents were required to answer all 17 questions in Waves I and II in order to be included in our analyzed sample. The final current depression index was standardized to the mean in order to facilitate model interpretation.

Siblings were classified as monozygotic twins (MZ), dizygotic twins (DZ), full siblings (FS), half siblings (HS), or cousins (CO), as indicated in the AddHealth data files.

Genotype: The 5-HTTLPR locus is characterized by a variable number of tandem repeat polymorphism with two predominant alleles that were assessed in the AddHealth study: the long $(l)$ allele with 16 repeats and the short $(s)$ allele with 14 repeats, the latter of which corresponds to a $\sim 44-\mathrm{bp}$ deletion in reference to the long allele. ${ }^{[28]}$ Respondents were assigned one of three possible 5-HTTLPR genotypes: homozygote long ( $l l$; referent category), homozygote short (ss), and heterozygote $(s l)$.

Age and race/ethnicity: Age was calculated using date of birth and date of interview and left as a continuous variable in the model. Race/ ethnicity was self-reported using the following categories: White (reference), African-American, Hispanic, Asian, and other race.

Family structure assessed the number of household resident parent(s) and categorized respondents as belonging to a twobiological parent family (referent category), a one-biological parent family (i.e. single biological parent or one biological parent and a stepparent), or "other family structure."

Family-level socioeconomic status (SES) was assessed by whether at least one resident parent was receiving public assistance (PA).

Social support was measured by averaging the responses to eight questions that represent respondents' perceived value and support from family members, friends, and teachers; responses ranged from 1 (not at all) to 5 (very much), with higher scores indicating more social support. If the respondents missed one or more of the eight questions, the average was determined from the remaining answered questions. The internal consistency of the eight social support questions used in this study was 0.78 .

County-level environment. Consistent with previous work, ${ }^{[29]} \mathrm{PA}$ was selected as a measure of exposure to poor social environments, i.e. a proxy for county-level deprivation. The proportion of households receiving PA income in each county for each respondent was assessed using US Census data from 1990, geocoded to respondents' interview data via the AddHealth contextual database. We calculated the median proportion of PA based on the counties represented by respondents in our data set and dummy variables were then created indicating 1 if the value is greater than the median and 0 otherwise. Individuals who relocated to a different county between Waves I and II were removed from the data set.

\section{STATISTICAL ANALYSIS}

A repeated multilevel modeling approach using mixed models was employed in our study, in which level 1 refers to the repeated measurements of individuals' depressive symptom scores (i.e. the scores obtained from the same individual at Wave I and Wave II), level 2 refers to the individual respondent, and level 3 refers to the family cluster to which the respondent belongs. The following equation describes the basic mixed model used in our analysis:

$$
\begin{gathered}
\mathrm{CESD}_{i j(s)}=\beta_{0}^{\prime} X_{i j}+\beta_{1}^{\prime} 5-H T T L P R_{i j}+\beta_{2}^{\prime} \text { family structure }_{i j}+\beta_{3}^{\prime} \mathrm{SES}_{i j}+\beta_{4} \text { support }_{i j}+\beta_{5} \\
\mathrm{PA}_{i j}+u_{j(s)}+v_{i j}+e_{i j(s)}
\end{gathered}
$$

where $i, j$ indicate individual and sibling cluster, respectively. Each beta represents a single coefficient or a vector of coefficients for each predictor component in the model; $X$ represents age and race, 5-HTTLPR represents the serotonin transporter promoter genotype, family structure represents the variants in resident parents, SES refers to household receipt of PA, support refers to social support, and PA refers to public assistance measured at the county level. The random effect of the family cluster is represented by $u_{j(s)}, v_{i j}$ is the random effect of the repeated observations on the same individual, and $e_{i j(s)}$ is the error term. This model allows the random effect of family cluster and the error term to vary by sibling type ${ }^{[30]}$ denoted by $s(s=\mathrm{MZ}$, DZ, FS, HS, and CO). All predictors were set at Wave I values and the outcome variable (depressive symptom score) was assessed as a repeated measure across Waves I and II, i.e. across a 1-year interval. Our first model tested the unadjusted (i.e. bivariate) associations between each separate covariate and the outcome. The second model predicted standardized depressive symptom scores adjusting for all the variables included in the model. Our third model again adjusted for all the covariates in the model and included a genotype $\times$ countylevel PA interaction term to assess potential $\mathrm{G} \times \mathrm{E}$ interactions between these two variables (using the $l l$ genotype and low PA as the referent categories). All models were stratified by gender, and all analyses were conducted using SAS v. 9.2, SAS Institute, Cary, NC.

\section{RESULTS}

Table 1 presents the descriptive statistics and unadjusted associations for the individual-, family- and county-level predictors included in our final model. The average age in both our male $(n=524)$ and female $(n=560)$ samples was approximately 16 years (range in males: $12-19$ years; range in females: $12-20$ years). The average depressive symptom score was significantly higher in female adolescents (27.8) versus male adolescents $(26.5 ; t=-3.55, P=.0004)$. Although a number of predictor variables also showed gender differences in unadjusted associations (Table 1), notable to this study was the detection of a significant protective effect of the $s l$ genotype in females and a significantly increased risk for depressive symptoms among males residing in high PA counties. When one sibling 
TABLE 1. Sample descriptives and unadjusted associations predicting standardized depressive symptom score, stratified by gender

\begin{tabular}{|c|c|c|c|c|c|c|c|c|c|c|c|c|}
\hline & \multicolumn{2}{|c|}{ Males $(n=524)$} & \multicolumn{2}{|c|}{ Females $(n=560)$} & \multicolumn{4}{|c|}{ Males $(n=524)$} & \multicolumn{4}{|c|}{ Females $(n=560)$} \\
\hline & $n /$ mean & $\% / s t d$ & $n /$ mean & $\% / s t d$ & $b$ & $P$ & $95 \%$ & \%CI & $b$ & $P$ & $95 \%$ & ${ }_{6} \mathrm{CI}$ \\
\hline \multicolumn{13}{|l|}{ Genotype } \\
\hline ss & 120 & 22.9 & 96 & 17.1 & .04 & .69 & -0.14 & 0.21 & .14 & .21 & -0.08 & 0.36 \\
\hline$s l$ & 244 & 46.6 & 265 & 47.3 & -.13 & .07 & -0.27 & 0.01 & -.16 & .04 & -0.32 & 0.00 \\
\hline$l l$ & 160 & 30.5 & 199 & 35.5 & .14 & .10 & -0.02 & 0.29 & .11 & .23 & -0.07 & 0.28 \\
\hline \multicolumn{13}{|l|}{ Demographics } \\
\hline Age & 16.1 & 1.6 & 16.0 & 1.7 & .04 & .05 & 0.00 & 0.09 & .02 & .44 & -0.03 & 0.06 \\
\hline White & 277 & 52.9 & 355 & 63.4 & -.36 & $<.0001$ & -0.52 & -0.20 & -.28 & $<.01$ & -0.47 & -0.10 \\
\hline Black & 82 & 15.6 & 76 & 13.6 & .28 & .02 & 0.05 & 0.50 & .10 & .46 & -0.16 & 0.35 \\
\hline Hispanic & 87 & 16.6 & 59 & 10.5 & .07 & .53 & -0.15 & 0.30 & .02 & .88 & -0.27 & 0.32 \\
\hline Asian & 44 & 8.4 & 28 & 5.0 & .36 & .02 & 0.07 & 0.65 & .58 & .01 & 0.14 & 1.01 \\
\hline Other & 34 & 6.5 & 42 & 7.5 & .26 & .12 & -0.07 & 0.59 & .44 & .02 & 0.09 & 0.80 \\
\hline \multicolumn{13}{|l|}{ Family structure } \\
\hline Two biological parents & 358 & 68.3 & 366 & 65.4 & -.26 & $<.01$ & -0.43 & -0.08 & -.33 & $<.001$ & -0.52 & -0.14 \\
\hline One biological parent & 150 & 28.6 & 171 & 30.5 & .25 & .01 & 0.07 & 0.44 & .33 & $<.001$ & 0.14 & 0.52 \\
\hline Other family structure & 16 & 3.1 & 23 & 4.1 & .13 & .60 & -0.35 & 0.61 & .13 & .55 & -0.30 & 0.56 \\
\hline \multicolumn{13}{|l|}{ SES and social support } \\
\hline Parent receives public assistance & 34 & 6.5 & 52 & 9.3 & .32 & .06 & -0.01 & 0.66 & .37 & .01 & 0.07 & 0.68 \\
\hline Social support & 4.0 & 0.5 & 4.0 & 0.6 & -.56 & $<.0001$ & -0.68 & -0.45 & -.75 & $<.0001$ & -0.86 & -0.63 \\
\hline \multicolumn{13}{|l|}{ County-level } \\
\hline High deprivation & 286 & 54.6 & 265 & 47.3 & .20 & .02 & 0.04 & 0.37 & .12 & .20 & -0.06 & 0.30 \\
\hline \multicolumn{13}{|l|}{ 17-item CES-D } \\
\hline Depressive Symptom Score & 26.5 & 5.6 & 27.8 & 6.4 & & & & & & & & \\
\hline
\end{tabular}

Significant effect estimates at $5 \%$ level are bold-faced, $b$ is the model parameter estimate, $P$ is the $P$-value, and CI is the paramater estimate confidence interval.

TABLE 2. Adjusted main effects model predicting standardized depressive symptom score, stratified by gender

\begin{tabular}{|c|c|c|c|c|c|c|c|c|}
\hline \multirow[b]{3}{*}{$s s$} & \multicolumn{4}{|c|}{ Male $(n=524)$} & \multicolumn{4}{|c|}{ Female $(n=560)$} \\
\hline & \multirow{2}{*}{$\begin{array}{c}b \\
-.09\end{array}$} & \multirow{2}{*}{$\begin{array}{c}P \\
.37\end{array}$} & \multicolumn{2}{|c|}{$95 \% \mathrm{CI}$} & \multirow{2}{*}{$\frac{b}{-.05}$} & \multirow{2}{*}{$\begin{array}{c}P \\
.66\end{array}$} & \multicolumn{2}{|c|}{$95 \% \mathrm{CI}$} \\
\hline & & & -0.28 & 0.10 & & & -0.26 & 0.17 \\
\hline$s l$ & -.12 & .13 & -0.27 & 0.03 & -.18 & .03 & -0.34 & -0.02 \\
\hline Age & .02 & .35 & -0.02 & 0.06 & -.01 & .63 & -0.05 & 0.03 \\
\hline Black/African-American & .33 & $<.01$ & 0.10 & 0.56 & .06 & .65 & -0.19 & 0.31 \\
\hline Hispanic & .21 & .05 & 0.00 & 0.41 & .13 & .32 & -0.13 & 0.38 \\
\hline Asian & .46 & $<.01$ & 0.18 & 0.74 & .68 & $<.001$ & 0.31 & 1.05 \\
\hline Other race & .31 & .05 & 0.00 & 0.61 & .23 & .13 & -0.07 & 0.54 \\
\hline One biological parent family & .09 & .30 & -0.08 & 0.26 & .27 & $<.01$ & 0.09 & 0.45 \\
\hline Other family structure & .10 & .64 & -0.33 & 0.54 & .09 & .65 & -0.30 & 0.48 \\
\hline Parent receives public assistance & .25 & .10 & -0.05 & 0.55 & .18 & .23 & -0.11 & 0.46 \\
\hline Social support & -.55 & $<.0001$ & -0.66 & -0.43 & -.74 & $<.0001$ & -0.85 & -0.62 \\
\hline High deprivation & .06 & .49 & -0.10 & 0.21 & .02 & .84 & -0.15 & 0.18 \\
\hline
\end{tabular}

Significant effect estimates at $5 \%$ level are bold-faced, $b$ is the model parameter estimate, $P$ is the $P$-value, and CI is the paramater estimate confidence interval.

per cluster was sampled randomly from each family, genotype frequencies for the 5-HTTLPR locus were in Hardy-Weinberg Equilibrium $\left(\chi^{2}=1.87, \quad d f=1\right.$ $P=.17)$. Consistent with previous work, ${ }^{[31]}$ the frequency of the $l l$ genotype was higher among black respondents (ll 50.6\%; sl 39.2\%; and ss $10.1 \%$ ) compared to whites (ll $31.3 \%$; sl $52.1 \%$; and ss $16.6 \%)$. Similarly, the frequency of $s s$ genotypes was higher among Asian (ll 11.1\%; sl 58.3\%; and ss 30.6\%) and Hispanic (ll 22.2\%; sl 50.0\%; and ss 27.8\%) respondents than among whites. Although these frequencies were statistically significantly different, genotype frequencies for all race/ethnic groups were in Hardy-Weinberg equilibrium. ${ }^{[32]}$

Table 2 presents the results of our multivariable, multilevel main effects model. For both males and females, higher social support was associated with significantly lower depressive symptom scores, and 
Asian race was associated with significantly higher depressive symptom scores. Among males only, belonging to any of the minority race/ethnic categories was a risk factor for significantly higher depressive symptom scores. Among females only, residing in a family in which there was only one biological parent was associated with significantly higher depressive symptom scores. In contrast, the $s l$ genotype showed significant protection against higher depressive symptom scores in this gender: holding all other predictors constant, there was an estimated -0.18 standard deviation change in the predicted mean depressive symptom scores of female $s l$ carriers $(b=-.18,95 \%$ CI: $-0.34,-0.02 ; P=.03)$.
Table 3 presents results from the multivariable models with the interaction term included. Among males, a significant interaction between county-level PA and 5-HTTLPR genotype was observed, such that males with the $s l$ genotype residing in counties with high PA were protected against higher depressive symptom scores $(b=-.32 ; 95 \%$ CI $-0.63,-0.02 ; P=.04)$. No significant interaction effects were observed among females.

Figure 1 presents the average depressive symptom scores for males and females, respectively, by 5-HTTLPR genotype and residence in high- versus low-deprivation counties, unadjusted for other covariates. Among males, the protective effect of the $s l$ genotype in high deprivation counties can be inferred

TABLE 3. Interaction effects model predicting standardized depresssive symptom score, stratified by gender

\begin{tabular}{|c|c|c|c|c|c|c|c|c|}
\hline \multirow[b]{3}{*}{ ss } & \multicolumn{4}{|c|}{ Male $(n=524)$} & \multicolumn{4}{|c|}{ Female $(n=560)$} \\
\hline & \multirow{2}{*}{$\begin{array}{c}b \\
.07\end{array}$} & \multirow{2}{*}{$\begin{array}{c}P \\
.64\end{array}$} & \multicolumn{2}{|c|}{$95 \% \mathrm{CI}$} & \multirow{2}{*}{$\frac{b}{-.08}$} & \multirow{2}{*}{$\begin{array}{c}P \\
.63\end{array}$} & \multicolumn{2}{|c|}{$95 \% \mathrm{CI}$} \\
\hline & & & -0.22 & 0.35 & & & -0.39 & 0.23 \\
\hline sl & .05 & .63 & -0.17 & 0.28 & -.10 & .37 & -0.32 & 0.12 \\
\hline Age & .02 & .34 & -0.02 & 0.06 & -.01 & .65 & -0.05 & 0.03 \\
\hline Black/African-American & .31 & .01 & 0.08 & 0.54 & .05 & .71 & -0.20 & 0.30 \\
\hline Hispanic & .21 & .05 & 0.00 & 0.41 & .12 & .36 & -0.14 & 0.37 \\
\hline Asian & .47 & $<.001$ & 0.20 & 0.75 & .68 & $<.001$ & 0.31 & 1.05 \\
\hline Other race & .30 & .05 & 0.00 & 0.60 & .22 & .15 & -0.08 & 0.53 \\
\hline One biological parent family & .09 & .29 & -0.08 & 0.26 & .28 & $<.01$ & 0.10 & 0.45 \\
\hline Other family structure & .09 & .69 & -0.35 & 0.52 & .08 & .69 & -0.31 & 0.47 \\
\hline Parent receives public assistance & .23 & .14 & -0.07 & 0.52 & .18 & .23 & -0.11 & 0.47 \\
\hline Social support & -.55 & $<.0001$ & -0.67 & -0.44 & -.74 & $<.0001$ & -0.86 & -0.63 \\
\hline High deprivation & .28 & .04 & 0.02 & 0.54 & .10 & .46 & -0.16 & 0.35 \\
\hline High deprivation $* s s$ & -.28 & .15 & -0.66 & 0.10 & .05 & .82 & -0.38 & 0.47 \\
\hline High deprivation $* s l$ & -.32 & .04 & -0.63 & -0.02 & -.19 & .25 & -0.50 & 0.13 \\
\hline
\end{tabular}

Significant effect estimates at $5 \%$ level are bold-faced, $b$ is the model parameter estimate, $P$ is the $P$-value, and CI is the parameter estimate confidence interval.
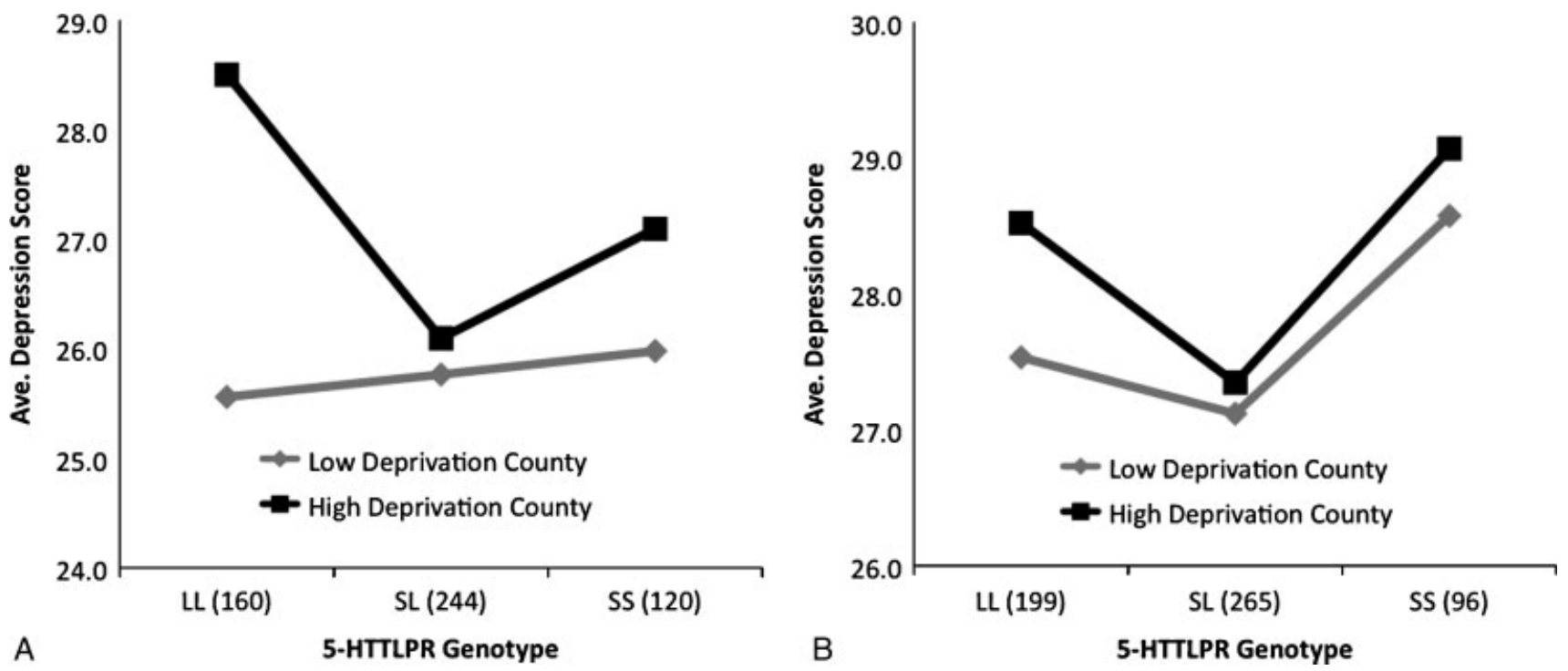

Figure 1. Average depressive symptom scores by 5-HTTLPR genotype and county-level deprivation, dichotomized as high versus low, among adolescent males (A) and females (B). 
from the noticeably lower depressive symptom scores compared to those observed for the ss or $l l$ genotypes; no similar difference is observed among males residing in counties with low deprivation. Among females, lower depressive symptoms scores are apparent among carriers of the $s l$ genotype irrespective of residence in high- or low-deprivation counties, consistent with the results obtained for this predictor in our unadjusted and multivariable main effect models (Tables 1 and 2). Depressive symptom scores were higher in female versus male adolescents for each genotype in each stratum; in low-deprivation counties, these results were statistically significant for the $l l$ and $s s$ genotypes $(t=-2.04, P=.04$ in both tests), and marginally significant for the $s l$ genotype $(t=-1.97, P=.06)$.

\section{DISCUSSION}

Our work confirms and extends the existing evidence for gender differences in the genetic and environmental determinants of adolescent depression and depressive symptoms. Using a genetic subsample of the National Longitudinal Study of Adolescent Health, we found that, in males, county-level environment modified the association between 5-HTTLPR genotype and depressive symptoms across a 1-year interval. No $\mathrm{G} \times \mathrm{E}$ associations were detected in adolescent females; however, in this group, there was evidence for a protective main effect of the $s l$ genotype in multivariable models. Taken together, these results represent the first report of a protective effect of the $s l$ genotype against depressive symptoms in adolescents that is differently manifested in males and females, and whose association with depressive symptoms in males is modified by a macro-level feature of the social environment.

Although the detection of the $s l$ genotype as protective has, to date, been detected in only one other study of adolescents of which we are aware, ${ }^{[17]}$ other studies have also presented results that are suggestive of a protective effect against depressive symptoms for this genotype in adults ${ }^{[33]}$ and children. ${ }^{[21]}$. In this study, our relatively large sample size may have enabled us to detect associations that other, more underpowered studies may have missed. Interestingly, in the aforementioned study of adolescents that detected a protective $s l$ effect and the analyses included what could be considered a measure of the macrosocial environment, i.e. residence in public, multifamily versus privately owned, single family housing; similar to the results presented in this study, depressive symptom scores were lowest only among adolescent males residing in multifamily housing and carrying the sl genotype. ${ }^{[17]}$ When studies have measured "E" via assessments of SLE or psychosocial stress, results have indicated that the $s$ allele confers a protective main effect against depressive symptoms in female adolescents [16] but is associated with increased depressive symptoms in this group when interacted with family-level environmental risks. ${ }^{[16,17]}$ Although these latter results have led some to suggest that adolescent boys may be protected from such "pathogenic" $\mathrm{G} \times \mathrm{E}$ interactions, ${ }^{[11]}$ it is also possible that current methods may be inadequate for measuring exposures and outcomes pertinent to this group. ${ }^{[11]}$

Although our goal in this work was not to address this last point, our working assumption that macrolevel environmental features may act as determinants of mental health may indeed have offered a salient lens through which to view $\mathrm{G} \times \mathrm{E}$ interactions relevant to depressive symptoms that might otherwise be missed among adolescent males. Importantly, our analyses detected an interaction between underlying genetic variability/vulnerability and county-level environment when controlling for potential family-level confounders (i.e. household receipt of $\mathrm{PA}$ ). That this $\mathrm{G} \times \mathrm{E}$ effect was detected only among males, in contrast to previous reports demonstrating a preponderance of $\mathrm{G} \times \mathrm{E}$ interactions among adolescent females when "E" is measured as SLE, suggests that different types of environmental measures may be salient for males and females of this age range; different environmental measures, in turn, may uncover $\mathrm{G} \times \mathrm{E}$ interactions at work with different alleles even at the same locus. More generally, these results suggest that among adolescents, macrosocial context may have differential effects by gender, such that adolescent males are more susceptible to contextual effects than their female counterparts. This suggestion is consistent with previous evaluations of neighborhood contextual effects on adolescent mental health. ${ }^{[23]}$ Similar findings have been reported in the twin studies literature, in which shared neighborhood-level environmental influences were found to be more important than genetic effects in determining antisocial behavior among adolescent males, whereas for adolescent girls, genetic factors were more important than shared environmental effects. ${ }^{[34]}$

From a population genetic perspective, one scenario that may account in part for the observations reported here is the signal of Tajima's $D$ surrounding the 5-HTTLPR locus. Tajima's $D$ is a statistical test that aims to distinguish between a DNA sequence evolving randomly ("neutrally") versus one evolving under a non-random process, such as directional selection or balancing selection. ${ }^{[35]}$ The University of Santa Cruz genome browser provides a snapshot of Tajima's $D$ values in three populations (in Americans of European, African, and Asian ancestry) estimated from the Perlgen data set. ${ }^{[36]}$ Tajima's $D$ can adopt values between -2 and +2 . High values of Tajima's $D$ generally indicate an excess of common variation in a region, which can be consistent with both balancing selection and population contraction.

In the area surrounding the genomic region assessed in this work (rs25531), the value of Tajima's $D$ is estimated to be quite high among individuals of European descent-near the maximum. This group was also 
the ethnic group with the greatest representation in our sample $(52.9 \%$ males and $63.4 \%$ females). If we assume that our sampled population is not contracting, then perhaps a scenario of balancing selection can offer insight into our previously unreported observation of a protective effect of the $s l$ genotype. Under this scenario, there would be a heterozygote advantagea "benefit" of having an $s l$ genotype that would be somewhat akin to the more commonly known sickle cell anemia example. As in the anemia example, the selective advantage of the $s l$ genotype may appear only under certain environmental conditions-in this case, adverse macrosocial conditions. Although this argument is inferential-Tajima's $D$ is assessed using nucleotide-level diversity, which was not directly assessed in the AddHealth genetic sample-and, furthermore, does not account for the protective main effect of the $s l$ genotype among adolescent females, the more general scenario of balancing selection might help to explain the heterogeneity of $\mathrm{G} \times \mathrm{E}$ results reported thus far for the 5-HTTLPR locus: maintenance of high levels of diversity at this locus may be advantageous in that different alleles and/or genotypes confer benefits to their bearers in different environmental contexts.

This study has a number of limitations that should be taken into consideration when evaluating its results. First, 5-HTTLPR genotype was assessed using a biallelic system, producing three possible genotypes for analysis in this work; other, relatively low-frequency alleles at this locus have been reported, ${ }^{[37]}$ including a novel $\mathrm{L}_{\mathrm{G}}$ allele thought to behave similarly to the more commonly occurring $s$ allele. ${ }^{[38,39]}$ Due to our reliance on secondary data, however, we were unable to assess their contribution in this work. Lack of consideration of the triallelic genotype, however, would have resulted in misclassification that would have reduced our power to detect genetic effects. Second, we observed significant racial/ethnic differences in 5-HTTLPR genotype frequencies and depressive symptoms, which raises the possibility that population stratification could have influenced our findings. However, we adjusted for selfreported race/ethnicity in our multivariable models (models 2 and 3), which has been shown to correspond well with ancestral classification using genetic markers. ${ }^{[40]}$ In addition, we reran all analyses in Whites only and findings were comparable, suggesting that population stratification cannot account for the results presented here.

Finally, there are likely additional environmental and molecular factors, at multiple levels, which contribute to depressive symptoms in adolescents that we were unable to capture in this study. For example, it is possible that including childhood maltreatment as an "E" variable in our models may have enabled us to detect depression-related $\mathrm{G} \times \mathrm{E}$ interactions among adolescent females consistent with previous reports in the literature ${ }^{[16]}$; in addition, evidence is emerging that epigenetic mechanisms may also play a role in regulating expression of the serotonin transporter gene, ${ }^{[41,42]}$ with one report indicating a nearly significant $(P=.07)$, higher methylation level of SLC6A4 in those without a lifetime history of major depression when compared to those who have at some point suffered from the disorder. ${ }^{[41]}$ Investigations focused on $\mathrm{G} \times \mathrm{E}$ interactions using the 5-HTTLPR locus are thus inherently complex and will likely require multiple levels of measurement at both the molecular and environmental levels to improve our identification of etiological risk factors for increased depressive symptoms.

Despite these limitations, this study confirms the need to investigate the determinants of depression separately in males and females, particularly in studies involving the 5-HTTLPR locus in adolescent populations. In addition, this study contributes to emergent literature suggesting that features of the macrosocial environment interact with individual genetic variation in the development of psychiatric disorders. ${ }^{[4]}$ Our results suggest that adolescent females with the 5-HTTLPR sl genotype are conferred protection against depressive symptoms independent of the larger social environment in which they reside; in contrast, among adolescent males, there is an evidence for a $\mathrm{G} \times \mathrm{E}$ interaction effect at the 5-HTTLPR locus, such that the $s l$ genotype confers protection against depression in males residing in adverse social environments. Future work should attempt to replicate the results presented in this study in other adolescent populations, and to more thoroughly investigate the distinct causal pathways that may link features of the social environment to risk for/resilience to depressive symptoms separately in males and females.

Acknowledgments. This research uses data from Add Health, a program project designed by J. Richard Udry, Peter S. Bearman, and Kathleen Mullan Harris, and funded by a grant P01-HD31921 from the Eunice Kennedy Shriver National Institute of Child Health and Human Development, with cooperative funding from 17 other agencies. Special acknowledgment to Ronald R. Rindfuss and Barbara Entwisle for their assistance in the original design. Persons interested in obtaining data files from Add Health should contact Add Health, Carolina Population Center, 123 W. Franklin Street, Chapel Hill, NC 27516-2524 (addhealth@unc.edu). No direct support was received from grant P01-HD31921 for this analysis.

\section{REFERENCES}

1. Rice F. The genetics of depression in childhood and adolescence. Curr Psychiatry Rep 2009;11:167-173.

2. Shih RA, Belmonte PL, Zandi PP. A review of the evidence from family, twin and adoption studies for a genetic contribution to adult psychiatric disorders. Int Rev Psychiatry 2004;16:260-283.

3. Rice F, Harold G, Thapar A. The genetic aetiology of childhood depression: a review. J Child Psychol Psychiatry 2002;43:65-79. 
4. Eley TC, Stevenson J. Exploring the covariation between anxiety and depression symptoms: a genetic analysis of the effects of age and sex. J Child Psychol Psychiatry 1999;40:1273-1282.

5. Silberg J, Pickles A, Rutter $M$ et al. The influence of genetic factors and life stress on depression among adolescent girls. Arch Gen Psychiatry 1999;56:225-232.

6. Moffitt TE, Caspi A, Rutter M. Strategy for investigating interactiosn between measured genes and measured environments. Arch Gen Psychiatry.2005;62:473-481.

7. Tao-Cheng JH, Zhou FC. Differential polarization of serotonin transporters in axons versus soma-dendrites: an immunogold electron microscopy study. Neuroscience 1999;94: 821-830.

8. Hariri AR, Weinberger DR. Functional neuroimaging of genetic variation in serotonergic neurotransmission. Genes Brain Behav 2003;2:341-349.

9. Lesch KP, Bengel D, Heils A et al. Association of anxiety-related traits with a polymorphism in the serotonin transporter gene regulatory region. Science 1996;274:1527-1531.

10. Caspi A, Sugden K, Moffitt TE et al. Influence of life stress on depression: moderation by a polymorphism in the 5-HTT gene. Science 2003;301:386-389.

11. Uher R, McGuffin P. The moderation by the serotonin transporter gene of environmental adversity in the aetiology of mental illness: review and methodological analysis. Mol Psychiatry 2008;13:131-146.

12. Risch $\mathrm{N}$, Herrell $\mathrm{R}$, Lehner $\mathrm{T}$ et al. Interaction between the serotonin transporter gene (5-HTTLPR), stressful life events, and risk of depression: a meta-analysis. JAMA 2009;301: 2462-2471.

13. Chipman P, Jorm AF, Prior M et al. No interaction between the serotonin transporter polymorphism (5-HTTLPR) and childhood adversity or recent stressful life events on symptoms of depression: results from two community surveys. Am J Med Genet B Neuropsychiatr Genet 2007;144B:561-565.

14. Laucht M, Treutlein J, Blomeyer D et al. Interaction between the 5-HTTLPR serotonin transporter polymorphism and environmental adversity for mood and anxiety psychopathology: evidence from a high-risk community sample of young adults. Int J Neuropsychopharmacol 2009:1-11.

15. Cicchetti D, Rogosch FA, Sturge-Apple ML. Interactions of child maltreatment and serotonin transporter and monoamine oxidase A polymorphisms: depressive symptomatology among adolescents from low socioeconomic status backgrounds. Dev Psychopathol 2007;19:1161-1180.

16. Eley TC, Sugden K, Corsico A et al. Gene-environment interaction analysis of serotonin system markers with adolescent depression. Mol Psychiatry 2004;9:908-915.

17. Sjoberg RL, Nilsson KW, Nordquist $\mathrm{N}$ et al. Development of depression: sex and the interaction between environment and a promoter polymorphism of the serotonin transporter gene. Int J Neuropsychopharmacol 2006;9:443-449.

18. Carlsson M, Carlsson A. A regional study of sex differences in rat brain serotonin. Prog Neuropsychopharmacol Biol Psychiatry 1988;12:53-61.

19. Barr CS, Newman TK, Schwandt M et al. Sexual dichotomy of an interaction between early adversity and the serotonin transporter gene promoter variant in rhesus macaques. Proc Natl Acad Sci USA 2004;101:12358-12363.

20. Jorm AF, Prior M, Sanson A et al. Association of a functional polymorphism of the serotonin transporter gene with anxietyrelated temperament and behavior problems in children: a longitudinal study from infancy to the mid-teens. Mol Psychiatry 2000;5:542-547.
21. Kaufman J, Yang BZ, Douglas-Palumberi H et al. Brain-derived neurotrophic factor-5-HTTLPR gene interactions and environmental modifiers of depression in children. Biol Psychiatry 2006;59:673-680.

22. Kaufman J, Yang BZ, Douglas-Palumberi H et al. Social supports and serotonin transporter gene moderate depression in maltreated children. Proc Natl Acad Sci USA 2004;101: 17316-17321.

23. Leventhal T, Brooks-Gunn J. Moving to opportunity: an experimental study of neighborhood effects on mental health. Am J Public Health 2003;93:1576-1582.

24. Kling JR, Jeffrey JB, Katz LF. Experimental analysis of neighborhood effects. Econometrica 2007;75:83-119.

25. Harris KM. The National Longitudinal Study of Adolescent Health (Add Health), Waves I \& II, 1994-1996; Wave III, 2001-2002 [machine-readable data file and documentation]. Chapell Hill, NC: Carolina Population Center, University of North Carolina at Chapel Hill; 2008.

26. Harris KM, Halpern CT, Smolen A et al. The National Longitudinal Study of Adolescent Health (Add Health) twin data. Twin Res Hum Genet 2006;9:988-997.

27. Radloff L. The CES-D scale: a self-report depression scale for research in the general population. Appl Psychol Meas 1977; 1:385-401.

28. Heils A, Teufel A, Petri S et al. Allelic variation of human serotonin transporter gene expression. J Neurochem 1996;66: 2621-2624.

29. Robert SA. Community-level socioeconomic status effects on adult health. J Health Soc Behav 1998;39:18-37.

30. Guo G, Wang J. The mixed or multilevel model for behavior genetic analysis. Behav Genet 2002;32:37-49.

31. Gelernter J, Kranzler H, Cubells JF. Serotonin transporter protein (SLC6A4) allele and haplotype frequencies and linkage disequilibria in African- and European-American and Japanese populations and in alcohol-dependent subjects. Hum Genet 1997;101:243-246.

32. Rodriguez S, Gaunt TR, Day IN. Hardy-Weinberg equilibrium testing of biological ascertainment for Mendelian randomization studies. Am J Epidemiol 2009;169:505-514.

33. Jacobs N, Kenis G, Peeters F et al. Stress-related negative affectivity and genetically altered serotonin transporter function: evidence of synergism in shaping risk of depression. Arch Gen Psychiatry 2006;63:989-996.

34. Tuvblad C, Grann M, Lichtenstein P. Heritability for adolescent antisocial behavior differs with socioeconomic status: geneenvironment interaction. J Child Psychol Psychiatry 2006;47: 734-743.

35. Tajima F. Statistical method for testing the neutral mutation hypothesis by DNA polymorphism. Genetics 1989;123: 585-595.

36. Hinds DA, Stuve LL, Nilsen GB et al. Whole-genome patterns of common DNA variation in three human populations. Science 2005;307:1072-1079.

37. Nakamura M, Ueno S, Sano A et al. The human serotonin transporter gene linked polymorphism (5-HTTLPR) shows ten novel allelic variants. Mol Psychiatry 2000;5:32-38.

38. Stein MB, Seedat S, Gelernter J. Serotonin transporter gene promoter polymorphism predicts SSRI response in generalized social anxiety disorder. Psychopharmacology (Berl) 2006;187: $68-72$.

39. Hu X, Oroszi G, Chun J et al. An expanded evaluation of the relationship of four alleles to the level of response to alcohol and the alcoholism risk. Alcohol Clin Exp Res 2005;29: 8-16. 
40. Tang H, Quertermous T, Rodriguez B et al. Genetic structure, self-identified race/ethnicity, and confounding in case-control association studies. Am J Hum Genet 2005;76:268-275.

41. Philibert RA, Sandhu H, Hollenbeck $\mathrm{N}$ et al. The relationship of 5HTT (SLC6A4) methylation and genotype on mRNA expression and liability to major depression and alcohol dependence in subjects from the Iowa Adoption Studies. Am J Med Genet B Neuropsychiatr Genet 2008;147B:543-549.
42. Philibert R, Madan A, Andersen A et al. Serotonin transporter mRNA levels are associated with the methylation of an upstream CpG island. Am J Med Genet B Neuropsychiatr Genet 2007;144B:101-105

43. Koenen KC, Aiello AE, Bakshis E et al. Modification of the association between serotonin transporter genotype and risk of posttraumatic stress disorder in adults by county-level social environment. Am J Epidemiol 2009;169:704-711. 